\title{
Reconstructing a Lattice Equation: \\ a Non-Autonomous Approach to the Hietarinta Equation
}

\author{
Giorgio GUBBIOTTI ${ }^{\dagger \ddagger}$ and Christian SCIMITERNA \\ $\dagger$ School of Mathematics and Statistics, F07, The University of Sydney, \\ New South Wales 2006, Australia \\ E-mail: giorgio.gubbiotti@sydney.edu.au \\ $¥$ Dipartimento di Matematica e Fisica, Università degli Studi Roma Tre \\ and Sezione INFN di Roma Tre, Via della Vasca Navale 84, 00146 Roma, Italy \\ E-mail: gubbiotti@mat.uniroma3.it,scimiterna@fis.uniroma3.it
}

Received April 30, 2017, in final form December 15, 2017; Published online January 09, 2018 https://doi.org/10.3842/SIGMA.2018.004

\begin{abstract}
In this paper we construct a non-autonomous version of the Hietarinta equation [Hietarinta J., J. Phys. A: Math. Gen. 37 (2004), L67-L73] and study its integrability properties. We show that this equation possess linear growth of the degrees of iterates, generalized symmetries depending on arbitrary functions, and that it is Darboux integrable. We use the first integrals to provide a general solution of this equation. In particular we show that this equation is a sub-case of the non-autonomous $Q_{\mathrm{V}}$ equation, and we provide a nonautonomous Möbius transformation to another equation found in [Hietarinta J., J. Nonlinear Math. Phys. 12 (2005), suppl. 2, 223-230] and appearing also in Boll's classification [Boll R., Ph.D. Thesis, Technische Universität Berlin, 2012].
\end{abstract}

Key words: quad-equations; Darboux integrability; algebraic entropy; generalized symmetries; exact solutions

2010 Mathematics Subject Classification: 37K10; 37K35; 37L20; 37L60; 39A14; 39A22

\section{Introduction}

Since its introduction the consistency around the cube (CAC) has been a source of many results in the classification of nonlinear integrable partial difference equations on a quad graph. The importance of this criterion relies on the fact that it ensures the existence of Bäcklund transformations [7, 11, 18, 45, 46] and, as a consequence, of Lax pairs. However [62] Lax pairs and Bäcklund transforms are associated with both linearizable and integrable equations. Let us point out that to be bona fide a Lax pair has to give rise to a genuine spectral problem [14], otherwise the Lax pair is a fake Lax pair $[12,13,15,35,36]$. A fake Lax pair is useless in proving (or disproving) the integrability, since it can be equally found for integrable and non-integrable equations. In the linearizable case Lax pairs are fake, even though proving this it is usually nontrivial [29].

The first attempt to classify all the multi-affine partial difference equations defined on the quad graph and possessing CAC was carried out in [1]. There the equation on the quad graph was treated as a geometric object not embedded in any $\mathbb{Z}^{2}$-lattice, as displayed in Fig. 1 . The quad-equation is an expression of the form

$$
Q\left(x, x_{1}, x_{2}, x_{12} ; \alpha_{1}, \alpha_{2}\right)=0,
$$

This paper is a contribution to the Special Issue on Symmetries and Integrability of Difference Equations. The full collection is available at http://www.emis.de/journals/SIGMA/SIDE12.html 
connecting some a priori independent fields $x, x_{1}, x_{2}, x_{12}$ assigned to the vertices of the quad graph, see Fig. 1. $Q$ is assumed to be a multi-affine polynomial in $x, x_{1}, x_{2}, x_{12}$ and, as shown in Fig. 1, $\alpha_{1}$ and $\alpha_{2}$ are parameters assigned to the edges of the quad graph.

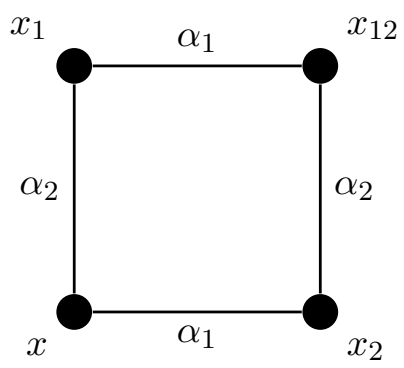

Figure 1. The purely geometric quad graph not embedded in any lattice.

In this setting, we define the consistency around the cube as follows: assume we are given six quad-equations

$$
\begin{aligned}
& A\left(x, x_{1}, x_{2}, x_{12} ; \alpha_{1}, \alpha_{2}\right)=0, \\
& \bar{A}\left(x_{3}, x_{13}, x_{23}, x_{123} ; \alpha_{1}, \alpha_{2}\right)=0, \\
& B\left(x, x_{2}, x_{3}, x_{23} ; \alpha_{2}, \alpha_{3}\right)=0, \\
& \bar{B}\left(x_{1}, x_{12}, x_{13}, x_{123} ; \alpha_{2}, \alpha_{3}\right)=0, \\
& C\left(x, x_{1}, x_{3}, x_{13} ; \alpha_{1}, \alpha_{3}\right)=0, \\
& \bar{C}\left(x_{2}, x_{12}, x_{23}, x_{123} ; \alpha_{1}, \alpha_{3}\right)=0,
\end{aligned}
$$

arranged on the faces of a cube as in Fig. 2. Using the system (1.2) we can compute $x_{12}, x_{23}$ and $x_{13}$ from (1.2a), (1.2c) and (1.2e) respectively. Then substituting these values into (1.2b), $(1.2 \mathrm{~d})$ and (1.2f) we have three different ways to compute $x_{123}$. If these three different ways of computing $x_{123}$ agree we say that the system (1.2) possesses the consistency around the cube.

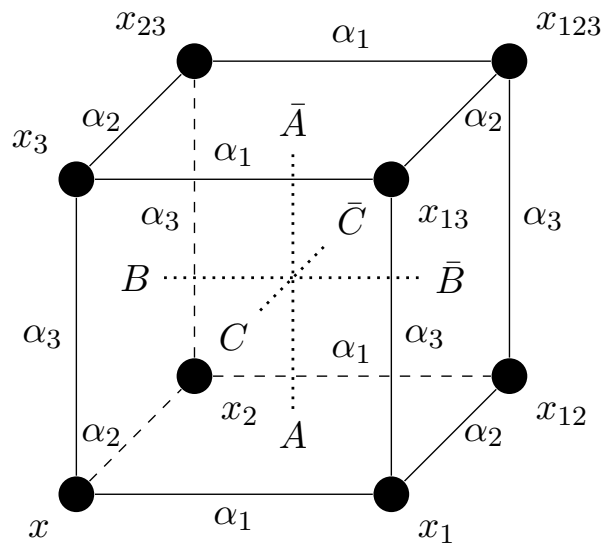

Figure 2. Equations on a cube.

In [1] the classification was carried out up to the action of a general Möbius transformation and up to point transformations of the edge parameters, with the additional assumptions:

1. All the faces of the cube in Fig. 2 carry the same equation up to the edge parameters. 
2. The quad-equation (1.1) possesses the $D_{4}$ discrete symmetries

$$
Q\left(x, x_{1}, x_{2}, x_{12} ; \alpha_{1}, \alpha_{2}\right)=\mu Q\left(x, x_{2}, x_{1}, x_{12} ; \alpha_{2}, \alpha_{1}\right)=\mu^{\prime} Q\left(x_{1}, x, x_{12}, x_{2} ; \alpha_{1}, \alpha_{2}\right),
$$

where $\mu, \mu^{\prime} \in\{ \pm 1\}$.

3. The system (1.2) possesses the tetrahedron property, i.e., $x_{123}$ is independent of $x$

$$
x_{123}=x_{123}\left(x, x_{1}, x_{2}, x_{3} ; \alpha_{1}, \alpha_{2}, \alpha_{3}\right) \Longrightarrow \frac{\partial x_{123}}{\partial x}=0 .
$$

The results were three classes of discrete autonomous equations with these properties: the $H$ equations, $Q$ equations and the $A$ equations. However the $A$ equations can be transformed in particular cases of the $Q$ equations through non-autonomous Möbius transformation. Therefore the $A$ equations are usually removed from the general classification.

After the introduction of the ABS equations J. Hietarinta tried to weaken the hypotheses of this classification. First in [37] he made a new search for new equations with no assumption about the symmetry and the tetrahedron property. Therein he obtained the following new equation

$$
\frac{x+e_{2}}{x+e_{1}} \frac{x_{12}+o_{2}}{x_{12}+o_{1}}=\frac{x_{1}+e_{2}}{x_{1}+o_{1}} \frac{x_{2}+o_{2}}{x_{2}+e_{1}},
$$

where $e_{i}$ and $o_{i}$ are constants. We will refer to this equation as the Hietarinta equation. It was later proved that the Hietarinta equation (1.4) embedded into a $\mathbb{Z}^{2}$-lattice with the standard embedding

$$
x \rightarrow u_{n, m}, \quad x_{1} \rightarrow u_{n+1, m}, \quad x_{2} \rightarrow u_{n, m+1}, \quad x_{12} \rightarrow u_{n+1, m+1},
$$

is linearizable [47]. In a subsequent paper [38] J. Hietarinta made a new classification adding the $D_{4}$ discrete symmetries (1.3) and he found three "new" equations, all linearizable.

Releasing the hypothesis that every face of the cube carried the same equation, in [2] were presented some new equations without classification purposes. A complete classification in this extended setting was then accomplished by Boll in a series of papers [8, 9], culminating in his Ph.D. Thesis [10]. In these papers the classification of all the consistent sextupletts of partial difference equations on the quad graph, i.e., systems of the form (1.2), has been carried out. The only technical assumption used in by Boll is the tetrahedron property. The obtained equations may fall into three disjoint families depending on their bi-quadratics

$$
h_{i j}=\frac{\partial Q}{\partial y_{k}} \frac{\partial Q}{\partial y_{l}}-Q \frac{\partial^{2} Q}{\partial y_{k} \partial y_{l}}, \quad Q=Q\left(y_{1}, y_{2}, y_{3}, y_{4} ; \alpha_{1}, \alpha_{2}\right),
$$

where we use a special notation for variables of $Q$, and the pair $\{k, l\}$ is the complement of the pair $\{i, j\}$ in $\{1,2,3,4\}$. A bi-quadratic is called degenerate if it contains linear factors of the form $y_{i}-c$, where $c$ is a constant, otherwise a bi-quadratic is called non-degenerate. The three families are characterized by how many bi-quadratics are degenerate:

- Q-type equations: all the bi-quadratics are non-degenerate,

- $H^{4}$-type equations: four bi-quadratics are degenerate,

- $H^{6}$-type equations: all of the six bi-quadratics are degenerate.

Let us notice that the $Q$ family is the same as the one introduced in [1]. The $H^{4}$ equations are divided into two subclasses: rhombic and trapezoidal, depending on their discrete symmetries.

We remark that the classification results of $[8,9,10]$ hold locally in the sense that they relate to a single quadrilateral cell or a single cube displayed in Figs. 1 and 2. The important problem 
of embedding these results into a two- or three-dimensional lattice, with preservation of the three-dimensional consistency condition, was discussed in $[2,61]$ by using the concept of a Black and White lattice. One way to solve this problem is to embed (1.1) into a $\mathbb{Z}^{2}$-lattice with an elementary cell of size greater than one. In this case, the quad-equation (1.1) can be extended to a lattice, and the lattice equation becomes integrable or linearizable. To this end, following $[8,9,10]$, we reflect the square with respect to the normal to its right and top sides and then complete a $2 \times 2$ lattice by again reflecting one of the obtained squares in the other direction. Such procedure is graphically described in Fig. 3.

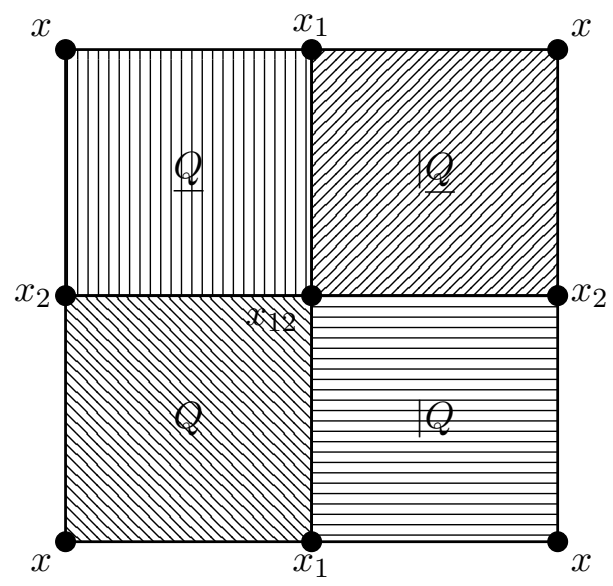

Figure 3. The "four stripe" lattice.

It corresponds to constructing three equations obtained from (1.1) by flipping its arguments

$$
\begin{aligned}
& Q=Q\left(x, x_{1}, x_{2}, x_{12} ; \alpha_{1}, \alpha_{2}\right)=0, \\
& \mid Q=Q\left(x_{1}, x, x_{12}, x_{2} ; \alpha_{1}, \alpha_{2}\right)=0, \\
& \underline{Q}=Q\left(x_{2}, x_{12}, x, x_{1} ; \alpha_{1}, \alpha_{2}\right)=0, \\
& \underline{Q}=Q\left(x_{12}, x_{2}, x_{1}, x ; \alpha_{1}, \alpha_{2}\right)=0 .
\end{aligned}
$$

By paving the whole $\mathbb{Z}^{2}$ with such equations, we get a partial difference equation which can be in principle studied using known methods. Since a priori $Q \neq|Q \neq Q \neq| Q$, the obtained lattice will be a four stripe lattice, i.e., an extension of the Black and White lattice considered in $[2,40,61]$. This gives rise to lattice equations with two-periodic coefficients for an unknown function $u_{n, m}$, with $(n, m) \in \mathbb{Z}^{2}$

$$
\begin{aligned}
F_{n}^{(+)} & F_{m}^{(+)} Q\left(u_{n, m}, u_{n+1, m}, u_{n, m+1}, u_{n+1, m+1} ; \alpha_{1}, \alpha_{2}\right) \\
& +F_{n}^{(-)} F_{m}^{(+)} \mid Q\left(u_{n, m}, u_{n+1, m}, u_{n, m+1}, u_{n+1, m+1} ; \alpha_{1}, \alpha_{2}\right) \\
& +F_{n}^{(+)} F_{m}^{(-)} \underline{Q}\left(u_{n, m}, u_{n+1, m}, u_{n, m+1}, u_{n+1, m+1} ; \alpha_{1}, \alpha_{2}\right) \\
& +F_{n}^{(-)} F_{m}^{(-)} \mid \underline{Q}\left(u_{n, m}, u_{n+1, m}, u_{n, m+1}, u_{n+1, m+1} ; \alpha_{1}, \alpha_{2}\right)=0,
\end{aligned}
$$

where

$$
F_{k}^{( \pm)}=\frac{1 \pm(-1)^{k}}{2}
$$

This explicit formula was first presented in [29]. We finally remark that the above construction can be carried out also at the level of the consistency cube displayed in Fig. 2 which is then embedded in a three dimensional lattice $\mathbb{Z}^{3}$ with coordinates $(n, m, p)$. The outcome of the 
procedure is a sextuplet in which $\bar{A}=T_{p} A, \bar{B}=T_{n} B$ and $\bar{C}=T_{m} C$ where $T_{n} h_{n, m, p}=h_{n+1, m, p}$, $T_{m} h_{n, m, p}=h_{n, m+1 . p}$ and $T_{p} h_{n, m, p}=h_{n, m, p+1}$ are translation operators. Therefore on the lattice the sextuplet becomes, as a matter of fact, a triplet of equations. For this reason when dealing with consistent equations embedded on the lattice we will speak about a triplet of equations. For more details on the construction of equations on the lattice from the single cell equations, we refer to the original papers [8, 9, 10,61], to the Appendix in [28] and to [26].

We remark that the construction outlined above can be applied to every consistent system of quad-equations (1.2) when one of the equations does not possess the $D_{4}$ discrete symmetries (1.3). Given an equation possessing the $D_{4}$ discrete symmetries (1.3), it can be shown that it reduces to standard embedding (1.5), see, e.g., [26, 28].

A detailed study of all the lattice equations derived from the rhombic $H^{4}$ family, including the construction of their three-leg forms, Lax pairs, Bäcklund transformations and infinite hierarchies of generalized symmetries, has been presented in [61]. There are plenty of results about the $Q$ and the rhombic $H^{4}$ equations. On the contrary, besides the CAC property little is known about the integrability features of the trapezoidal $H^{4}$ equations and of the $H^{6}$ equations. These equations where thoroughly studied in a series of papers [28, 29, 30,31,32, 33, 34] with some unexpected results. In [28] the algebraic entropy [6, 39, 58, 59] of the trapezoidal $H^{4}$ and the $H^{6}$ equations was computed. The result of this computation showed that the rate of growth of all the trapezoidal $H^{4}$ and of all $H^{6}$ equations is linear. According to the algebraic entropy conjecture $[19,39]$ this fact implies the linearizability. In [34], following the suggestions obtained in $[29,30]$, it was showed that the trapezoidal $H^{4}$ equations and all the $H^{6}$ equations are Darboux integrable [3]. Finally in [33] it was shown, applying a modification of the procedure presented in [21], that Darboux integrability provides the general solutions of these equations. Moreover in [30] it was showed that the three quad-equations found in [38] were Darboux integrable.

In this paper we study a new non-autonomous version of the Hietarinta equation (1.4) obtained using the prescriptions of $[2,8,9,10,61]$. We then show that this equation possesses properties analogous to those of the other three equations considered in [38].

First to construct this new equation we start from the polynomial version of the Hietarinta equation (1.4)

$$
Q=\left(x+e_{2}\right)\left(x_{1}+o_{1}\right)\left(x_{2}+e_{1}\right)\left(x_{12}+o_{2}\right)-\left(x+e_{1}\right)\left(x_{1}+e_{2}\right)\left(x_{2}+o_{2}\right)\left(x_{12}+o_{1}\right) .
$$

This equation does not possess the $D_{4}$ discrete symmetries (1.3), then in addition to the standard embedding (1.5) we can consider the non-autonomous embedding given by formula (1.7). Applying formula (1.7) to the Hietarinta equation in polynomial form (1.9) we obtain the following non-autonomous lattice equation

$$
\begin{aligned}
& \left(u_{n, m}+K_{n, m}^{(1)}\right)\left(u_{n+1, m}+K_{n+1, m}^{(1)}\right)\left(u_{n, m+1}+K_{n, m+1}^{(1)}\right)\left(u_{n+1, m+1}+K_{n+1, m+1}^{(1)}\right) \\
& \quad=\left(u_{n, m}+K_{n, m}^{(2)}\right)\left(u_{n+1, m}+K_{n+1, m}^{(2)}\right)\left(u_{n, m+1}+K_{n, m+1}^{(2)}\right)\left(u_{n+1, m+1}+K_{n+1, m+1}^{(2)}\right),
\end{aligned}
$$

which is similar to the original equation (1.9), but with the four constants $e_{i}, o_{i}$ replaced by the following two two-periodic functions of the lattice variables

$$
\begin{aligned}
& K_{n, m}^{(1)}=F_{n}^{(+)} F_{m}^{(+)} e_{2}+F_{n}^{(-)} F_{m}^{(+)} o_{1}+F_{n}^{(+)} F_{m}^{(-)} e_{1}+F_{n}^{(-)} F_{m}^{(-)} o_{2}, \\
& K_{n, m}^{(2)}=F_{n}^{(+)} F_{m}^{(+)} e_{1}+F_{n}^{(-)} F_{m}^{(+)} e_{2}+F_{n}^{(+)} F_{m}^{(-)} o_{2}+F_{n}^{(-)} F_{m}^{(-)} o_{1} .
\end{aligned}
$$

We will call equation (1.10) the non-autonomous Hietarinta equation. We remark that the non-autonomous equation (1.10) depends on the same number of parameters as its autonomous counterpart (1.9). Then it is easy to show that the non-autonomous Hietarinta equation (1.10) does not reduce to the autonomous one (1.9) for special values of the parameters. As a matter of fact equation (1.10) is not a non-autonomous extension of the Hietarinta equation, but it is 
a different embedding of the same single-cell equation (1.9) which produces a non-autonomous dynamical system.

Following the procedure outlined in $[8,9,10]$ and using the notations of Appendix A in [28] we now explain how to obtain the consistency around the cube for the non-autonomous Hietarinta equation (1.10) and discuss its properties. First we denote by

$$
\widehat{Q}_{n, m}=\widehat{Q}_{n, m}\left(u_{n, m}, u_{n+1, m}, u_{n, m+1}, u_{n+1, m+1} ; e_{1}, o_{1}, e_{2}, o_{2}\right)
$$

the difference between the left- and the right-hand side in (1.10). As explained above we add a third direction $p$ to the field $u_{n, m}$

$$
u_{n, m} \rightarrow u_{n, m, p}
$$

Then from the sextuplet presented in [37] and the construction in $[8,9,10,28]$ we have the following triplet of equations

$$
\begin{aligned}
& A=\widehat{Q}_{n, m, p}\left(u_{n, m, p}, u_{n+1, m, p}, u_{n, m+1, p}, u_{n+1, m+1, p} ; e_{1}, o_{1}, e_{2}, o_{2}\right), \\
& B=\widehat{Q}_{n, m, p}\left(u_{n, m, p}, u_{n, m+1, p}, u_{n, m, p+1}, u_{n, m+1, p+1} ; e_{2}, o_{2}, e_{3}, o_{3}\right), \\
& C=\widehat{Q}_{n, m, p}\left(u_{n, m, p}, u_{n+1, m, p}, u_{n, m, p+1}, u_{n+1, m, p+1} ; e_{1}, o_{1}, e_{3}, o_{3}\right),
\end{aligned}
$$

being $\bar{A}=T_{p} A, \bar{B}=T_{n} B$ and $\bar{C}=T_{m} C$ as remarked above. We emphasize that the explicit dependence on the $p$ variable is introduced in (1.11) though the two-periodic embedding in $\mathbb{Z}^{3}[10,28]$. In practice this corresponds to the replace $F_{n}^{( \pm)} F_{m}^{( \pm)}$with $F_{n}^{( \pm)} F_{m}^{( \pm)} F_{p}^{( \pm)}$coherently with the embedding. Consistency can be checked directly: the value of $u_{n+1, m+1, p+1}$ is given by

$$
\begin{aligned}
u_{n+1, m+1, p+1}= & F_{n}^{(+)} F_{m}^{(+)} F_{p}^{(+)} \frac{N_{[123]}}{D_{[123]}}+\left.F_{n}^{(-)} F_{m}^{(+)} F_{p}^{(+)} \frac{N_{[123]}}{D_{[123]}}\right|_{e_{1} \leftrightarrow o_{1}} \\
& +\left.F_{n}^{(+)} F_{m}^{(-)} F_{p}^{(+)} \frac{N_{[123]}}{D_{[123]}}\right|_{e_{2} \leftrightarrow o_{2}}+\left.F_{n}^{(+)} F_{m}^{(+)} F_{p}^{(-)} \frac{N_{[123]}}{D_{[123]}}\right|_{e_{3} \leftrightarrow o_{3}} \\
& +\left.F_{n}^{(-)} F_{m}^{(-)} F_{p}^{(+)} \frac{N_{[123]}}{D_{[123]}}\right|_{\substack{e_{2} \leftrightarrow o_{1} \\
e_{2} \leftrightarrow o_{2}}}+\left.F_{n}^{(-)} F_{m}^{(+)} F_{p}^{(-)} \frac{N_{[123]}}{D_{[123]}}\right|_{\substack{e_{1} \leftrightarrow o_{1} \\
e_{3} \leftrightarrow o_{3}}} \\
& +\left.F_{n}^{(+)} F_{m}^{(-)} F_{p}^{(-)} \frac{N_{[123]}}{D_{[123]}}\right|_{\substack{e_{2} \leftrightarrow o_{2} \\
e_{3} \leftrightarrow o_{3}}}+\left.F_{n}^{(-)} F_{m}^{(-)} F_{p}^{(-)} \frac{N_{[123]}}{D_{[123]}}\right|_{\substack{e_{1} \leftrightarrow o_{1} \\
e_{2} \leftrightarrow o_{2} \\
e_{3} \leftrightarrow o_{3}}},
\end{aligned}
$$

where

$$
\begin{aligned}
& N_{[123]}=N_{[123]}\left(u_{n, m, p}, u_{n+1, m, p}, u_{n, m+1, p}, u_{n, m, p+1} ; e_{1}, e_{2}, e_{3}, o_{1}, o_{2}, o_{3}\right), \\
& D_{[123]}=D_{[123]}\left(u_{n, m, p}, u_{n+1, m, p}, u_{n, m+1, p}, u_{n, m, p+1} ; e_{1}, e_{2}, e_{3}, o_{1}, o_{2}, o_{3}\right),
\end{aligned}
$$

are given as in [37] by

$$
\begin{aligned}
N_{[123]}= & -u_{n, m, p}\left(u_{n+1, m, p}+o_{1}\right)\left(u_{n, m+1, p}+o_{2}\right)\left(u_{n, m, p+1}+o_{3}\right)\left(o_{1}-o_{2}\right)\left(o_{2}-o_{3}\right)\left(o_{3}-o_{1}\right) \\
& +\left(u_{n+1, m, p}+o_{1}\right)\left(u_{n, m+1, p}+o_{2}\right)\left(u_{n, m, p+1}+o_{3}\right) \\
& \times\left[\left(e_{1} e_{2}+e_{3} o_{3}\right) o_{3}\left(o_{1}-o_{2}\right)+\left(e_{2} e_{3}+e_{1} o_{1}\right) o_{1}\left(o_{2}-o_{3}\right)+\left(e_{3} e_{1}+e_{2} o_{2}\right) o_{2}\left(o_{3}-o_{1}\right)\right] \\
& +\left(u_{n, m, p}+e_{3}\right)\left(u_{n+1, m, p}+o_{1}\right)\left(u_{n, m+1, p}+o_{2}\right) o_{3}\left(o_{1}-o_{2}\right)\left(e_{2}-o_{3}\right)\left(o_{3}-e_{1}\right) \\
& +\left(u_{n, m, p}+e_{1}\right)\left(u_{n, m+1, p}+o_{2}\right)\left(u_{n, m, p+1}+o_{3}\right) o_{1}\left(o_{2}-o_{3}\right)\left(e_{3}-o_{1}\right)\left(o_{1}-e_{2}\right) \\
& +\left(u_{n, m, p}+e_{2}\right)\left(u_{n, m, p+1}+o_{3}\right)\left(u_{n+1, m, p}+o_{1}\right) o_{2}\left(o_{3}-o_{1}\right)\left(e_{1}-o_{2}\right)\left(o_{2}-e_{3}\right),
\end{aligned}
$$




$$
\begin{aligned}
D_{[123]}= & \left(u_{n+1, m, p}+o_{1}\right)\left(u_{n, m+1, p}+o_{2}\right)\left(u_{n, m, p+1}+o_{3}\right) \\
& \times\left[\left(e_{1} e_{2}+e_{3} o_{3}\right)\left(o_{2}-o_{1}\right)+\left(e_{2} e_{3}+e_{1} o_{1}\right)\left(o_{3}-o_{2}\right)+\left(e_{3} e_{1}+e_{2} o_{2}\right)\left(o_{1}-o_{3}\right)\right] \\
& +\left(u_{n, m, p}+e_{3}\right)\left(u_{n+1, m, p}+o_{1}\right)\left(u_{n, m+1, p}+o_{2}\right)\left(o_{1}-o_{2}\right)\left(e_{1}-o_{3}\right)\left(e_{2}-o_{3}\right) \\
& +\left(u_{n, m, p}+e_{1}\right)\left(u_{n, m+1, p}+o_{2}\right)\left(u_{n, m, p+1}+o_{3}\right)\left(o_{2}-o_{3}\right)\left(e_{2}-o_{1}\right)\left(e_{3}-o_{1}\right) \\
& +\left(u_{n, m, p}+e_{2}\right)\left(u_{n, m, p+1}+o_{3}\right)\left(u_{n+1, m, p}+o_{1}\right)\left(o_{3}-o_{1}\right)\left(e_{3}-o_{2}\right)\left(e_{1}-o_{2}\right),
\end{aligned}
$$

and the notation $\left.A\right|_{p \leftrightarrow q}$ means that the two parameters must be exchanged. It is clear that $u_{n+1, m+1, p+1}$ as given by (1.12) depends explicitly on $u_{n, m, p}$, so that the triplet (1.11) does not posses the tetrahedron property.

For the rest of this paper we will make a complete study of the non-autonomous Hietarinta equation (1.10). In Section 2 we will show that equation (1.10) possesses linear growth in all directions. In Section 3 we identify equation (1.10) with a sub-case of the non-autonomous $Q_{\mathrm{V}}$ equation [32]. Then we show that the equation (1.10) can be reduced to the quad-equation

$$
v_{n, m} v_{n+1, m+1}+v_{n+1, m} v_{n, m+1}=0 .
$$

which was discussed in $[37,38]$. This finding immediately explains the linearizability property of this equation and it is the main result of this paper. Indeed equation (1.13) is linked to the discrete wave equation

$$
w_{n+1, m+1}-w_{n+1, m}-w_{n, m+1}+w_{n, m}=0
$$

through the non-autonomous, transcendental transformation

$$
v_{n, m}=\left[F_{m}^{(+)}+F_{m}^{(-)}\left(F_{n}^{(+)}-F_{n}^{(-)}\right)\right] e^{w_{n, m}} .
$$

This result is of course quite unexpected. Then we briefly discuss the rôle of the tetrahedron property in these investigations. In the subsequent Sections we treat the equation (1.10) into detail in order to show all the interesting properties that were found for the trapezoidal $H^{4}$ and $H^{6}$ equations $[28,29,31,32,34,33]$ with this simple example. In Section 4 we will prove that equation (1.10) is Darboux integrable with first order first integrals. In Section 5 we will present the generalized symmetries of the non-autonomous Hietarinta equation (1.10) of every order by using the first integrals and the so called symmetry drivers [53]. In Section 6 we use the first integrals to derive the general solution of equation (1.10). In Section 7 we give some conclusion and we give an outlook on presented results.

The main objective of this paper is to show how the Hietarinta equation (1.4), behaves under the Boll's embedding, becoming then the non-autonomous equation (1.10) and then to discuss the property of this new embedding. In this sense our starting point is similar to those adopted in [40], where it was shown that different consistent embeddings can produce equations with different properties.

\section{Algebraic entropy}

Algebraic entropy is as a test of integrability for discrete systems. Given a bi-rational map, which can be an ordinary difference equation, a differential difference equation or even a partial difference equation, the basic idea is to examine the growth of the degree of its iterates, and extract a canonical quantity, which is an index of complexity of the map. This canonical quantity will be the algebraic entropy (or its avatar the dynamical degree) $[6,16,19,50,57]$. In $[56,58]$ the method was developed in the case of quad-equations and then used as a classifying tool [39]. A slight generalization of the method was given in [28] where it was showed that non-autonomous equations can have more that a single sequence of degrees. 
Given a sequence of degrees obtained iterating a rational map

$$
1, d_{0}, d_{1}, \ldots, d_{k}, \ldots,
$$

we define the algebraic entropy of this map to be

$$
\eta=\lim _{k \rightarrow \infty} \frac{1}{k} \log d_{k}
$$

This quantity is canonical as it is invariant with respect to bi-rational transformations. To give a practical example, the transformation (1.15) which maps equation (1.13) into the discrete wave equation (1.14) does not preserve a priori algebraic entropy, because it is not bi-rational, see [24]. Geometrically algebraic entropy is deeply linked with the structure of the singularities of a discrete system, and in some cases it can be computed resorting to this structure $[17,51,55,60]$.

Instead of computing the whole sequence of iterates which is clearly impossible, only a finite number of iterates is computed. Then some tools like generating functions [42] or discrete derivatives are used in order to obtain the asymptotic behaviour of the series [23, 25].

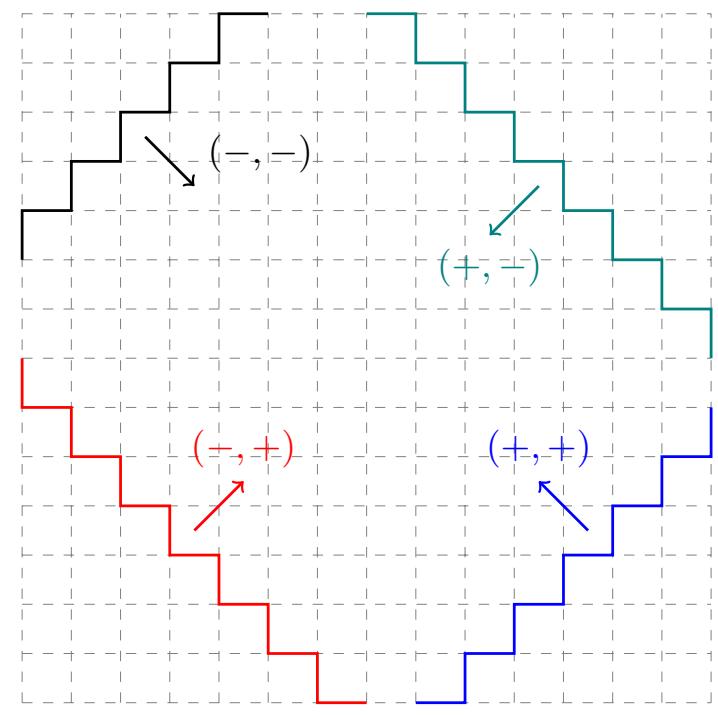

Figure 4. Principal growth directions.

The classification of lattice equations based on the algebraic entropy test is:

linear growth: the equation is linearizable,

polynomial growth: the equation is integrable,

exponential growth: the equation is chaotic.

We have performed the algebraic entropy analysis in the principal growth directions [58], shown in Fig. 4, of the non-autonomous Hietarinta equation (1.10) to identify its behaviour. To this end we used the SymPy [54] module ae2d.py [26, 27]. We found that the non-autonomous Hietarinta equation (1.10) is isotropic, i.e., its sequence of degrees is the same in every direction, and despite being non-autonomous and two-periodic it possess a single sequence of degrees given by

$$
1,3,5,7,9,11,13, \ldots
$$

The sequence (2.2) is asymptotically (in this case exactly) fitted by

$$
d_{k}=2 k+1, \quad \forall k \in \mathbb{N},
$$


and the algebraic entropy from (2.1) is clearly zero. Since the growth is linear we expect the non-autonomous Hietarinta equation (1.10) to be linearizable. In the next section we will show why the linearization arises.

\section{Point transformations and tetrahedron property}

In [32] it was proved that there exists a non-autonomous, two-periodic generalization of the $Q_{\mathrm{V}}$ equation [59] given by

$$
\begin{aligned}
& a_{1} u_{n, m} u_{n+1, m} u_{n, m+1} u_{n+1, m+1} \\
& \quad+\left[a_{2,0}-(-1)^{n} a_{2,1}-(-1)^{m} a_{2,2}+(-1)^{n+m} a_{2,3}\right] u_{n, m} u_{n, m+1} u_{n+1, m+1} \\
& \quad+\left[a_{2,0}+(-1)^{n} a_{2,1}-(-1)^{m} a_{2,2}-(-1)^{n+m} a_{2,3}\right] u_{n+1, m} u_{n, m+1} u_{n+1, m+1} \\
& \quad+\left[a_{2,0}+(-1)^{n} a_{2,1}+(-1)^{m} a_{2,2}+(-1)^{n+m} a_{2,3}\right] u_{n, m} u_{n+1, m} u_{n+1, m+1} \\
& \quad+\left[a_{2,0}-(-1)^{n} a_{2,1}+(-1)^{m} a_{2,2}-(-1)^{n+m} a_{2,3}\right] u_{n, m} u_{n+1, m} u_{n, m+1} \\
& \quad+\left[a_{3,0}-(-1)^{m} a_{3,2}\right] u_{n, m} u_{n+1, m}+\left[a_{3,0}+(-1)^{m} a_{3,2}\right] u_{n, m+1} u_{n+1, m+1} \\
& \quad+\left[a_{4,0}-(-1)^{n+m} a_{4,3}\right] u_{n, m} u_{n+1, m+1}+\left[a_{4,0}+(-1)^{n+m} a_{4,3}\right] u_{n+1, m} u_{n, m+1} \\
& \quad+\left[a_{5,0}-(-1)^{n} a_{5,1}\right] u_{n+1, m} u_{n+1, m+1}+\left[a_{5,0}+(-1)^{n} a_{5,1}\right] u_{n, m} u_{n, m+1} \\
& \quad+\left[a_{6,0}+(-1)^{n} a_{6,1}-(-1)^{m} a_{6,2}-(-1)^{n+m} a_{6,3}\right] u_{n, m} \\
& \quad+\left[a_{6,0}-(-1)^{n} a_{6,1}-(-1)^{m} a_{6,2}+(-1)^{n+m} a_{6,3}\right] u_{n+1, m} \\
& \quad+\left[a_{6,0}+(-1)^{n} a_{6,1}+(-1)^{m} a_{6,2}+(-1)^{n+m} a_{6,3}\right] u_{n, m+1} \\
& \quad+\left[a_{6,0}-(-1)^{n} a_{6,1}+(-1)^{m} a_{6,2}-(-1)^{n+m} a_{6,3}\right] u_{n+1, m+1}+a_{7}=0 .
\end{aligned}
$$

In [32] it has been checked heuristically that this equation is integrable according to the algebraic entropy test, possessing quadratic growth of the degrees of the iterates, as was later rigorously shown using the factorization approach in [49]. Additionally in [32] it was proved that equation (3.1) contains as particular cases all the equations coming from the Boll's classifications, i.e., the rhombic $H^{4}$ equations, the trapezoidal $H^{4}$ equations, the $H^{6}$ equations and the $Q$ equations. Upon the substitution $a_{2,1}=a_{2,2}=a_{2,3}=a_{3,2}=a_{4,3}=a_{5,1}=a_{6,1}=a_{6,2}=a_{6,3}=0$ the non-autonomous $Q_{\mathrm{V}}$ equation reduces to the original $Q_{\mathrm{V}}$ equation presented in [59]. For this reasons equation (3.1) has been called the non-autonomous, or two-periodic, $Q_{\mathrm{V}}$ equation.

It is just a matter of computation to show that the original autonomous Hietarinta equation (1.4) is not a sub-case of the autonomous $Q_{\mathrm{V}}$ equation, but that the non-autonomous Hietarinta equation is a sub-case of the non-autonomous $Q_{\mathrm{V}}$ equation (3.1) with the following values of the coefficients

$$
\begin{aligned}
& a_{1}=0, \\
& a_{2,0}=0, \quad a_{2,1}=e_{2}-o_{2}, \quad a_{2,2}=e_{1}-o_{1}, \quad a_{2,3}=e_{1}+o_{1}-o_{2}-e_{2}, \\
& a_{3,0}=-\left(e_{2}-o_{2}\right)\left(e_{1}-o_{1}\right), \quad a_{3,2}=-\left(e_{2}+o_{2}\right)\left(e_{1}-o_{1}\right), \\
& a_{4,0}=0, \quad a_{4,3}=2\left(e_{2} o_{2}-o_{1} e_{1}\right), \\
& a_{5,0}=\left(e_{2}-o_{2}\right)\left(e_{1}-o_{1}\right), \quad a_{5,1}=-\left(e_{2}-o_{2}\right)\left(o_{1}+e_{1}\right), \\
& a_{6,0}=0, \quad a_{6,1}=-o_{1} e_{1}\left(e_{2}-o_{2}\right), \quad a_{6,2}=-\left(e_{1}-o_{1}\right) e_{2} o_{2}, \\
& a_{6,3}=o_{2} e_{1} o_{1}+e_{2} o_{1} e_{1}-e_{2} o_{2} e_{1}-e_{2} o_{2} o_{1}, \\
& a_{7}=0 .
\end{aligned}
$$

In the Introduction we discussed how the non-autonomous Hietarinta equation (1.10) comes from the triplet (1.11) which does not possess the tetrahedron property. However equation (3.2) shows that equation (1.10) is a particular case of the non-autonomous $Q_{\mathrm{V}}$ equation (3.1) and 
non-autonomous $Q_{\mathrm{V}}$ equation (3.1) contains all the equations of Boll's classification. This, along with the result of the algebraic entropy obtained in Section 2, suggests that a transformation mapping equation (1.10) into an equation of Boll's classification might exist. As stated before all the equations from Boll's classification come from sextuplet (triplet on the lattice) possessing the tetrahedron property, therefore the existence of such transformation will prove that the same equation can come from a different sextuplet (triplet on the lattice) possessing tetrahedron property. This turns out to be true since it can be shown that the non-autonomous, two-periodic Möbius transformation

$$
u_{n, m}=-\frac{\left[e_{1} F_{n}^{(+)}-o_{1} F_{n}^{(-)}\left(F_{m}^{(+)}-F_{m}^{(-)}\right)\right] v_{n, m}+e_{2} F_{m}^{(+)}+o_{2} F_{m}^{(-)}}{\left[\left(F_{n}^{(+)}-F_{n}^{(-)}\right) F_{m}^{(+)}+F_{m}^{(-)}\right] v_{n, m}+1}
$$

maps the non-autonomous Hietarinta equation (1.10) into equation (1.13). The transformation (3.3) can be interpreted at level of single cells as a simultaneous transformation of all the variables associated to the vertices $x, x_{1}, x_{2}$ and $x_{12}$, i.e., acting with an element of the group Möb $^{4}[1,5,28]$. Following the explicit formula given in [28] the element of Möb ${ }^{4}$ corresponding to $(3.3)$ is given by

$$
x=-\frac{e_{1} X+e_{2}}{X+1}, \quad x_{1}=-\frac{o_{1} X_{1}-e_{2}}{X_{1}-1}, \quad x_{2}=-\frac{e_{1} X_{2}+o_{2}}{X_{2}+1}, \quad x_{12}=-\frac{o_{1} X_{12}+o_{2}}{X_{12}-1} .
$$

This brings the Hietarinta equation on the single cell (1.9) into the single cell version of (1.13)

$$
X X_{12}+X_{1} X_{2}=0 .
$$

We now discuss what the existence of the transformation (3.3) implies at the level of the consistency around the cube. To make the discussion easier to follow, here will work with the equation on the single cell (1.9). We will show that allowing a simultaneous transformation of all the points $x, x_{1}, x_{2}, x_{3}, x_{12}, x_{13}, x_{23}$ and $x_{123}$, i.e., by using the group Möb ${ }^{8}$ it is possible to produce a sextuplet of consistent quad-equations of which (1.13) is the base equation. Indeed let us start from the sextuple of equations for the (1.4) equation given in [37]

$$
\begin{aligned}
A & =\frac{x+e_{2}}{x+e_{1}} \frac{x_{12}+o_{2}}{x_{12}+o_{1}}-\frac{x_{1}+e_{2}}{x_{1}+o_{1}} \frac{x_{2}+o_{2}}{x_{2}+e_{1}}, \\
\bar{A} & =\frac{x_{3}+e_{2}}{x_{3}+e_{13}} \frac{x_{123}+o_{2}}{x_{123}+o_{1}}-\frac{x_{13}+e_{2}}{x_{1}+o_{1}} \frac{x_{23}+o_{2}}{x_{23}+e_{1}}, \\
B & =\frac{x+e_{3}}{x+e_{2}} \frac{x_{23}+o_{3}}{x_{23}+o_{2}}-\frac{x_{2}+e_{3}}{x_{2}+o_{2}} \frac{x_{3}+o_{3}}{x_{3}+e_{2}}, \\
\bar{B} & =\frac{x_{1}+e_{3}}{x_{1}+e_{2}} \frac{x_{123}+o_{3}}{x_{123}+o_{2}}-\frac{x_{2}+e_{3}}{x_{12}+o_{2}} \frac{x_{13}+o_{3}}{x_{13}+e_{2}}, \\
C & =\frac{x+e_{3}}{x+e_{1}} \frac{x_{13}+o_{3}}{x_{13}+o_{1}}-\frac{x_{1}+e_{3}}{x_{1}+o_{1}} \frac{x_{3}+o_{3}}{x_{3}+e_{1}}, \\
\bar{C} & =\frac{x_{2}+e_{3}}{x_{2}+e_{1}} \frac{x_{123}+o_{3}}{x_{123}+o_{1}}-\frac{x_{12}+e_{3}}{x_{12}+o_{1}} \frac{x_{23}+o_{3}}{x_{23}+e_{1}} .
\end{aligned}
$$

Then the Möb ${ }^{8}$ transformation given by

$$
\begin{aligned}
& x=-\frac{e_{1} X+e_{2}}{X+1}, \quad x_{1}=-\frac{o_{1} X_{1}-e_{2}}{X_{1}-1}, \quad x_{2}=-\frac{e_{1} X_{2}+o_{2}}{X_{2}+1}, \quad x_{3}=-\frac{e_{1} X_{3}+e_{2}}{X_{3}+1}, \\
& x_{12}=-\frac{o_{1} X_{12}+o_{2}}{X_{12}+1}, \quad x_{13}=-\frac{o_{1} X_{13}-e_{2}}{X_{13}-1}, \quad x_{23}=-\frac{e_{1} X_{23}+o_{2}}{X_{23}+1}, \\
& x_{123}=-\frac{o_{1} X_{123}+o_{2}}{X_{123}+1}
\end{aligned}
$$


brings the system (3.5) into the following one

$$
\begin{aligned}
A= & X X_{12}+X_{1} X_{2}, \\
\bar{A}= & X_{3} X_{123}+X_{13} X_{23}, \\
B= & {\left[\left(e_{2}-e_{3}\right)\left(e_{1}-o_{3}\right) X_{3}-X\left(e_{2}-o_{3}\right)\left(e_{1}-e_{3}\right)\right] X_{2} X_{23} } \\
& +\left(e_{3}-o_{2}\right)\left[\left(e_{1}-o_{3}\right) X_{3}+e_{2}-o_{3}\right] X X_{23} \\
& +\left(o_{2}-o_{3}\right)\left(\left(e_{1}-e_{3}\right) X+e_{2}-e_{3}\right) X_{2} X_{3}, \\
\bar{B}= & {\left[\left(o_{1}-o_{3}\right)\left(e_{2}-e_{3}\right) X_{13}+\left(e_{3}-o_{1}\right)\left(e_{2}-o_{3}\right) X_{1}\right] X_{12} X_{123} } \\
& -\left(e_{3}-o_{2}\right)\left[\left(o_{1}-o_{3}\right) X_{13}-e_{2}+o_{3}\right] X_{1} X_{123} \\
& -\left(o_{2}-o_{3}\right)\left[\left(o_{1}-e_{3}\right) X_{1}-e_{2}+e_{3}\right] X_{13} X_{12}, \\
C= & e_{3} o_{3}\left[\left(X_{3}+1\right) X_{1}-(X+1) X_{13}-X+X_{3}\right] \\
& +e_{3}\left[\left(X-X_{1}\right) e_{2}-X_{3}\left(X_{1}+1\right) e_{1}+o_{1}(X+1) X_{13}\right] \\
& +o_{3}\left[\left(e_{2}+e_{1} X\right) X_{13}+e_{1} X-e_{2} X_{3}-o_{1} X_{1}\left(X_{3}+1\right)\right] \\
& -o_{1}\left(e_{2}+e_{1} X\right) X_{13}+e_{1}\left[e_{2}\left(X_{3}-X_{1}\right)+o_{1} X_{1} X_{3}\right]+o_{1} e_{2} X_{1}, \\
\bar{C}= & e_{3}\left[\left(X_{2}+1\right) X_{123}-\left(1+X_{23}\right) X_{12}-X_{23}+X_{2}\right] o_{3} \\
& +e_{3}\left[\left(o_{2}+e_{1} X_{23}\right) X_{12}-o_{1}\left(X_{2}+1\right) X_{123}+e_{1} X_{23}-o_{2} X_{2}\right] \\
& +o_{3}\left[o_{1}\left(X_{23}+1\right) X_{12}-\left(o_{2}+e_{1} X_{2}\right) X_{123}+o_{2} X_{23}-e_{1} X_{2}\right] \\
& +o_{1}\left(o_{2}+e_{1} X_{2}\right) X_{123}-o_{1}\left(o_{2}+e_{1} X_{23}\right) X_{12}+e_{1} o_{2}\left(X_{2}-X_{23}\right) .
\end{aligned}
$$

It is easy to show that the system (3.7) do not possess the tetrahedron property. Indeed computing $X_{123}$ we obtain

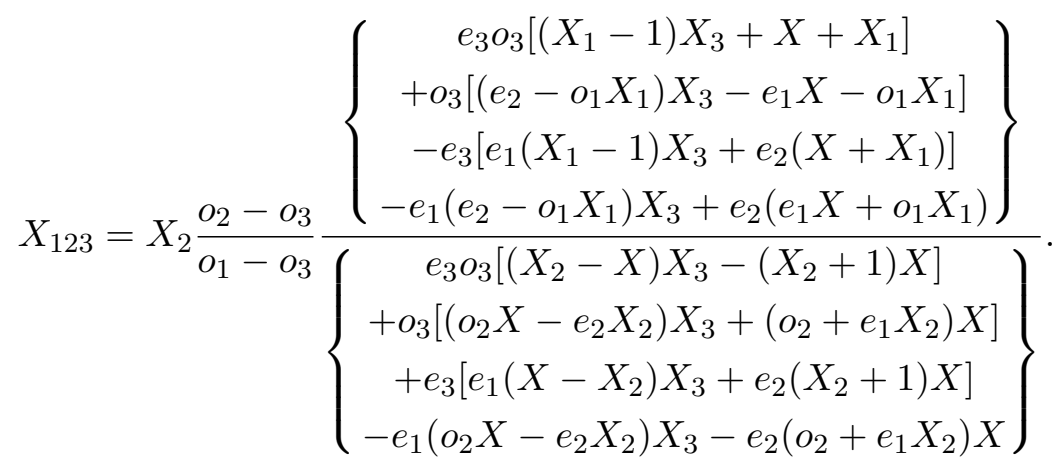

Remark 3.1. We remark that the use of $\mathrm{Möb}^{4}$ and $\mathrm{Möb}^{8}$ transformations like (3.4) and (3.6) is naturally allowed since we are in the framework of the non-autonomous CAC construction given in $[2,8,9,10]$. Non-autonomous Möbius transformations can be used also in the autonomous case, see [1,38], but in non-autonomous case they are the natural group which preserves the classification [28].

On the other hand equation (1.13) is a particular case of the ${ }_{1} D_{4}$ equation from $[8,9,10,28]$

$$
\begin{aligned}
& \delta_{1}\left(F_{n}^{(-)} v_{n, m} v_{n, m+1}+F_{n}^{(+)} v_{n+1, m} v_{n+1, m+1}\right)+\delta_{2}\left(F_{m}^{(-)} v_{n, m} v_{n+1, m}+F_{m}^{(+)} v_{n, m+1} v_{n+1, m+1}\right) \\
& \quad+v_{n, m} v_{n+1, m+1}+v_{n+1, m} v_{n, m+1}+\delta_{3}=0
\end{aligned}
$$

with $\delta_{1}=\delta_{2}=\delta_{3}=0$. This means that the non-autonomous Hietarinta equation (1.10) is not an independent equation, but is part of Boll's classification.

In particular this implies that equation (1.13) can arise also as bottom equation of a triplet possessing tetrahedron property. The relevant triplet is that coming from Case D in Theorem 3.12 in [10] that in our notation reads as

$$
A=v_{n, m, p} v_{n+1, m+1, p}+v_{n+1, m, p} v_{n, m+1, p},
$$




$$
\begin{aligned}
& B=\lambda\left(v_{n, m, p} v_{n, m+1, p}+v_{n, m, p+1} v_{n, m+1, p+1}\right)-v_{n, m, p} v_{n, m+1, p+1}-v_{n, m+1, p} v_{n, m, p+1}, \\
& C=\frac{1}{\lambda}\left(v_{n, m, p} v_{n+1, m, p}+v_{n, m, p+1} v_{n+1, m, p+1}\right)-v_{n, m, p} v_{n+1, m, p+1}-v_{n+1, m, p} v_{n, m, p+1},
\end{aligned}
$$

Here $\lambda$ is a new non-zero parameter. The triplet (3.9) is consistent and possesses the tetrahedron property since

$$
v_{n+1, m+1, p+1}=-\frac{v_{n+1, m, p} v_{n, m+1, p}}{v_{n, m, p+1}} .
$$

Remark 3.2. We recall that the equation (1.13) can be derived from another triplet without the tetrahedron property. As it was discussed in [37, 38] the equation (1.13) can thought as $A$ equation of the following triplet (we are already on the lattice)

$$
\begin{aligned}
& A=v_{n, m, p} v_{n+1, m+1, p}+v_{n+1, m, p} v_{n, m+1, p}, \\
& B=v_{n, m, p} v_{n, m+1, p+1}+v_{n, m+1, p} v_{n, m, p+1}, \\
& C=v_{n, m, p} v_{n+1, m, p+1}+v_{n+1, m, p} v_{n, m, p+1},
\end{aligned}
$$

which is obtained from (1.13) properly permuting the shifted variables. The triplet (3.10) is consistent on the cube but with no tetrahedron property since

$$
v_{n+1, m+1, p+1}=-\frac{v_{n+1, m, p} v_{n, m+1, p} v_{n, m, p+1}}{v_{n, m}^{2}} .
$$

This last result is clearly unexpected. A priori it would have been very difficult to find out that the non-autonomous version of the Hietarinta equation (1.4) was in fact a particular case of the ${ }_{1} D_{4}$ equation (3.8), since the first one arises from the triplet (1.11) without tetrahedron property, whereas the latter one arises from the triplet (3.10) with tetrahedron property. However, in general, a quad-equation can arise both from a sextuplet (triplet) with or without the tetrahedron property since the tetrahedron property is a property of the sextuplet (triplet) of equations and not a property of the equation itself. Examples of this phenomenon were already known in literature $[2,4,40]$. The example presented here is another one and it is rather non-trivial.

We stress out that since the non-autonomous Hietarinta equation (1.10) is linearizable its Lax pairs obtained both from triplet (1.11) and from reversing the transformation (3.3) in the triplet (3.9) should both be fake. Being fake these two Lax pair cannot give much information. However also integrable equations can have, in principle, Lax pairs coming from sextuplet (triplet) without tetrahedron property. Given a quad-equation with such properties it will be an interesting issue to investigate the relationship between these Lax pairs as it was done for the continuous Painlevé I and II equations [41]. We remark that in this case we are ensured that there is no point transformation between the sextuplet (3.5) and the triplet (3.7) since the first one does not possess the tetrahedron property while the second one does. On the other hand it is possible to prove the triplet (1.11) and the triplet (3.10) are not related by a Möb ${ }^{8}$ transformation even though they both do not possess the tetrahedron property.

Remark 3.3. We observe also that the Möbius transformation

$$
y_{n, m}=\frac{u_{n, m}+K_{n, m}^{(1)}}{u_{n, m}+K_{n, m}^{(2)}}
$$

bring equation (1.10) into another autonomous quad-equation

$$
y_{n, m} y_{n+1, m} y_{n, m+1} y_{n+1, m+1}=1 \text {. }
$$


This other equation is linked with equation (1.13) through the non-autonomous Möbius transformation [38]

$$
y_{n, m}=\left(F_{n}^{(+)} F_{m}^{(+)}+F_{n}^{(-)} F_{m}^{(-)}\right) v_{n, m}+\frac{F_{n}^{(-)} F_{m}^{(+)}+F_{n}^{(+)} F_{m}^{(-)}}{v_{n, m}} .
$$

The existence of the point transformations (3.3) and (3.11) is in itself sufficient to explain the linearizability of the non-autonomous Hietarinta equation (1.10), since equation (1.13) is linearizable, Darboux integrable and its generalized symmetries of every order are known [30]. These properties can then be inferred through this point transformation, but for the rest of this paper we have chosen to present a direct derivation. Our choice is motivated from the fact that equation (1.10) can be seen as a simple, yet nontrivial, example of all the properties found in the study of the trapezoidal $H^{4}$ and $H^{6}$ equations.

\section{Darboux integrability}

Suppose we are given a quad-equation, possibly non-autonomous

$$
Q_{n, m}\left(u_{n, m}, u_{n+1, m}, u_{n, m+1}, u_{n+1, m+1}\right)=0 .
$$

We say that such a quad-equation is Darboux integrable if there exist two independent first integrals, one containing only shifts in $n$, and the other containing only shifts in $m$, i.e., that there exist two independent functions

$$
\begin{aligned}
& W_{1}=W_{1, n, m}\left(u_{n+l_{1}, m}, u_{n+l_{1}+1, m}, \ldots, u_{n+k_{1}, m}\right), \\
& W_{2}=W_{2, n, m}\left(u_{n, m+l_{2}}, u_{n, m+l_{2}+1}, \ldots, u_{n, m+k_{2}}\right),
\end{aligned}
$$

where $l_{1}<k_{1}$ and $l_{2}<k_{2}$ are integers, such that the relations

$$
\begin{aligned}
& \left(T_{n}-\mathrm{Id}\right) W_{2}=0, \\
& \left(T_{m}-\mathrm{Id}\right) W_{1}=0,
\end{aligned}
$$

where $T_{n} h_{n, m}=h_{n+1, m}, T_{m} h_{n, m}=h_{n, m+1}$, and $\operatorname{Id} h_{n, m}=h_{n, m}$, hold true identically on the solutions of (4.1). The number $k_{i}-l_{i}$, where $i=1,2$, is called the order of the first integral $W_{i}$.

Any Darboux integrable equation is linearizable [3]. Indeed let us introduce two new fields $\tilde{u}_{n, m}=W_{1, n, m}$ and $\hat{u}_{n, m}=W_{2, n, m}$. Then

$$
\begin{aligned}
& u_{n, m} \rightarrow \tilde{u}_{n, m}, \\
& u_{n, m} \rightarrow \hat{u}_{n, m},
\end{aligned}
$$

define two non-point transformations of the field $u_{n, m}$ into $\tilde{u}_{n, m}$ and $\hat{u}_{n, m}$. Moreover these two new fields satisfy two trivial linear equations

$$
\begin{aligned}
& \tilde{u}_{n, m+1}-\tilde{u}_{n, m}=0, \\
& \hat{u}_{n+1, m}-\hat{u}_{n, m}=0 .
\end{aligned}
$$

Therefore we can conclude that any Darboux integrable equation is linearizable in two different ways, i.e., using transformation (4.3a) bringing to (4.4a) or using the transformation (4.3b) bringing to $(4.4 \mathrm{~b})$. 
Methods for calculating first integrals of non-autonomous quad-equations (4.1) with twoperiodic coefficients were given in [22, 34]. In particular in [34] was presented a new algorithm that relies on the fact that in the case of non-autonomous quad-equations (4.1) with two-periodic coefficients we can, in general, represent the first integrals in the form

$$
W_{i}=F_{n}^{(+)} F_{m}^{(+)} W_{i}^{(+,+)}+F_{n}^{(-)} F_{m}^{(+)} W_{i}^{(-,+)}+F_{n}^{(+)} F_{m}^{(-)} W_{i}^{(+,-)}+F_{n}^{(-)} F_{m}^{(-)} W_{i}^{(-,-)},
$$

where $F_{k}^{( \pm)}$are given by $(1.8)$ and the $W_{i}^{( \pm, \pm)}$are functions.

Applying the algorithm presented in [34] we find that the non-autonomous Hietarinta equation (1.10) possesses two first order first integrals in both directions

$$
\begin{aligned}
& W_{1}=\alpha_{1} W_{1}^{\left(\alpha_{1}\right)}+\beta_{1} W_{1}^{\left(\beta_{1}\right)}, \\
& W_{2}=\alpha_{2} W_{2}^{\left(\alpha_{2}\right)}+\beta_{2} W_{2}^{\left(\beta_{2}\right)},
\end{aligned}
$$

where

$$
\begin{aligned}
& W_{1}^{\left(\alpha_{1}\right)}=F_{n}^{(+)} F_{m}^{(+)} \frac{\left(u_{n, m}+e_{2}\right)\left(u_{n+1, m}+o_{1}\right)}{\left(u_{n, m}+e_{1}\right)\left(u_{n+1, m}+e_{2}\right)}+F_{n}^{(+)} F_{m}^{(-)} \frac{\left(u_{n, m}+o_{2}\right)\left(u_{n+1, m}+o_{1}\right)}{\left(u_{n, m}+e_{1}\right)\left(u_{n+1, m}+o_{2}\right)}, \\
& W_{1}^{\left(\beta_{1}\right)}=F_{n}^{(-)} F_{m}^{(+)} \frac{\left(u_{n, m}+o_{1}\right)\left(u_{n+1, m}+e_{2}\right)}{\left(u_{n, m}+e_{2}\right)\left(u_{n+1, m}+e_{1}\right)}+F_{n}^{(-)} F_{m}^{(-)} \frac{\left(u_{n, m}+o_{1}\right)\left(o_{2}+u_{n+1, m}\right)}{\left(u_{n, m}+o_{2}\right)\left(u_{n+1, m}+e_{1}\right)}, \\
& W_{2}^{\left(\alpha_{2}\right)}=F_{n}^{(+)} F_{m}^{(+)} \frac{\left(u_{n, m}+e_{2}\right)\left(u_{n, m+1}+e_{1}\right)}{\left(u_{n, m}+e_{1}\right)\left(u_{n, m+1}+o_{2}\right)}+F_{n}^{(-)} F_{m}^{(+)} \frac{\left(u_{n, m}+e_{2}\right)\left(o_{1}+u_{n, m+1}\right)}{\left(u_{n, m}+o_{1}\right)\left(u_{n, m+1}+o_{2}\right)}, \\
& W_{2}^{\left(\beta_{2}\right)}=F_{n}^{(+)} F_{m}^{(-)} \frac{\left(u_{n, m}+o_{2}\right)\left(u_{n, m+1}+e_{1}\right)}{\left(u_{n, m}+e_{1}\right)\left(u_{n, m+1}+e_{2}\right)}+F_{n}^{(-)} F_{m}^{(-)} \frac{\left(u_{n, m}+o_{2}\right)\left(u_{n, m+1}+o_{1}\right)}{\left(u_{n, m}+o_{1}\right)\left(u_{n, m+1}+e_{2}\right)} .
\end{aligned}
$$

\section{Generalized symmetries}

A vector field of the form

$$
\hat{X}=g_{n, m}\left(\mathbf{u}_{n, m}^{D}\right) \partial_{u_{n, m}}, \quad \mathbf{u}_{n, m}^{D}=\left\{u_{n+i, m+j}\right\}_{i=l_{1}, \ldots, k_{1}, j=l_{2}, \ldots, k_{2}},
$$

where $l_{1}<k_{1}$ and $l_{2}<k_{2}$ is said to be a generalized symmetry for the quad-equation (4.1) if its discrete prolongation

$$
\operatorname{pr}^{D} \hat{X}=g_{n, m} \partial_{u_{n, m}}+T_{n} g_{n, m} \partial_{u_{n+1, m}}+T_{m} g_{n, m} \partial_{u_{n, m+1}}+T_{n} T_{m} g_{n, m} \partial_{u_{n+1, m+1}},
$$

is such that

$$
g_{n, m} \frac{\partial Q_{n, m}}{\partial u_{n, m}}+T_{n} g_{n, m} \frac{\partial Q_{n, m}}{\partial u_{n+1, m}}+T_{m} g_{n, m} \frac{\partial Q_{n, m}}{\partial u_{n, m+1}}+T_{n} T_{m} g_{n, m} \frac{\partial Q_{n, m}}{\partial u_{n+1, m+1}}=0,
$$

identically on the solutions of (4.1) [20]. Symmetries of this kind with $k_{i}=-l_{i}=1$, i.e., threepoint generalized symmetries were first considered in [48]. Moreover three-point generalized symmetries were threated in [43, 44], and the general case was discussed in [20]. In [20] it was also proved that quad-equations (4.1) do not posses "mixed" symmetries, i.e., the function $g_{n, m}$ in (5.1), known as the characteristic of the generalized symmetry, is the sum of two simpler functions depending only on variables shifted only in one direction

$$
g_{n, m}\left(\mathbf{u}_{n, m}^{D}\right)=g_{n, m}^{(1)}\left(u_{n+l_{i}, m}, \ldots, u_{n+k_{1}, m}\right)+g_{n, m}^{(2)}\left(u_{n, m+l_{2}}, \ldots, u_{n, m+k_{2}}\right) .
$$

The relationship between generalized symmetries and Darboux integrability is well known in the continuous case [63] and in the discrete case have been investigated in [3, 52, 53]. As 
conjectured in [30] and proved in [53], a quad-equation is Darboux integrable if and only if it possesses generalized symmetries depending on arbitrary function in the following form

$$
\begin{aligned}
& g_{n, m}^{(1)}=R^{(1)}\left(F_{n}\left(T_{n}^{p_{1}} W_{1}, \ldots, T_{n}^{q_{1}} W_{1}\right)\right), \\
& g_{n, m}^{(2)}=R^{(2)}\left(G_{m}\left(T_{m}^{p_{2}} W_{2}, \ldots, T_{m}^{q_{2}} W_{2}\right)\right),
\end{aligned}
$$

where $p_{i}<q_{i}$ and $R^{(i)}$ are operators of the form

$$
\begin{aligned}
& R^{(1)}=\sum_{r=j_{1}}^{h_{1}} \lambda_{r}\left(u_{n+l_{i}^{\prime}, m}, \ldots, u_{n+k_{1}^{\prime}, m}\right) T_{n}^{r}, \\
& R^{(2)}=\sum_{r=j_{2}}^{h_{2}} \mu_{r}\left(u_{n, m+l_{2}^{\prime}}, \ldots, u_{n, m+k_{2}^{\prime}}\right) T_{m}^{r},
\end{aligned}
$$

with $j_{i}<h_{i}$ and $l_{i}^{\prime}<k_{i}^{\prime}$. The operators $R^{(i)}(5.3)$ are called symmetry drivers.

It is then easy to show that the non-autonomous Hietarinta equation possesses the following two-point generalized symmetries, i.e., such that $k_{i}=1, l_{i}=0$ in (5.1)

$$
\begin{gathered}
g_{n, m}^{(1)}=\left[F_{n}^{(+)} F_{m}^{(+)} \frac{\left(u_{n, m}+e_{1}\right)\left(u_{n, m}+e_{2}\right)}{e_{1}-e_{2}}+F_{n}^{(+)} F_{m}^{(-)} \frac{\left(u_{n, m}+e_{1}\right)\left(u_{n, m}+o_{2}\right)}{e_{1}-o_{2}}\right] F_{n}^{(1)}\left(W_{1}^{\left(\alpha_{1}\right)}\right) \\
+\left[F_{n}^{(-)} F_{m}^{(+)} \frac{\left(u_{n, m}+e_{2}\right)\left(u_{n, m}+o_{1}\right)}{e_{2}-o_{1}}-F_{n}^{(-)} F_{m}^{(-)} \frac{\left(u_{n, m}+o_{2}\right)\left(o_{1}+u_{n, m}\right)}{o_{1}-o_{2}}\right] G_{n}^{(1)}\left(W_{1}^{\left(\beta_{1}\right)}\right), \\
g_{n, m}^{(2)}=\left[F_{n}^{(+)} F_{m}^{(+)} \frac{\left(u_{n, m}+e_{1}\right)\left(u_{n, m}+e_{2}\right)}{e_{1}-e_{2}}-F_{n}^{(-)} F_{m}^{(+)} \frac{\left(u_{n, m}+e_{2}\right)\left(u_{n, m}+o_{1}\right)}{e_{2}-o_{1}}\right] F_{m}^{(2)}\left(W_{2}^{\left(\alpha_{1}\right)}\right) \\
+\left[F_{n}^{(+)} F_{m}^{(-)} \frac{\left(u_{n, m}+e_{1}\right)\left(u_{n, m}+o_{2}\right)}{\left(e_{1}-o_{2}\right)}+F_{n}^{(-)} F_{m}^{(-)} \frac{\left(u_{n, m}+o_{2}\right)\left(u_{n, m}+o_{1}\right)}{o_{1}-o_{2}}\right] G_{m}^{(2)}\left(W_{2}^{\left(\alpha_{1}\right)}\right),
\end{gathered}
$$

where $F_{k}^{(i)}$ and $G_{k}^{(i)}$ are arbitrary functions of their arguments. This implies that we have two multipliticative symmetry drivers $R^{(i)}(5.3)$ in each direction

$$
\begin{aligned}
& R^{\left(1, \alpha_{1}\right)}=F_{n}^{(+)} F_{m}^{(+)} \frac{\left(u_{n, m}+e_{1}\right)\left(u_{n, m}+e_{2}\right)}{e_{1}-e_{2}}+F_{n}^{(+)} F_{m}^{(-)} \frac{\left(u_{n, m}+e_{1}\right)\left(u_{n, m}+o_{2}\right)}{e_{1}-o_{2}}, \\
& R^{\left(1, \beta_{1}\right)}=F_{n}^{(-)} F_{m}^{(+)} \frac{\left(u_{n, m}+e_{2}\right)\left(u_{n, m}+o_{1}\right)}{e_{2}-o_{1}}-F_{n}^{(-)} F_{m}^{(-)} \frac{\left(u_{n, m}+o_{2}\right)\left(o_{1}+u_{n, m}\right)}{o_{1}-o_{2}}, \\
& R^{\left(2, \alpha_{2}\right)}=F_{n}^{(+)} F_{m}^{(+)} \frac{\left(u_{n, m}+e_{1}\right)\left(u_{n, m}+e_{2}\right)}{e_{1}-e_{2}}-F_{n}^{(-)} F_{m}^{(+)} \frac{\left(u_{n, m}+e_{2}\right)\left(u_{n, m}+o_{1}\right)}{e_{2}-o_{1}}, \\
& R^{\left(2, \beta_{2}\right)}=F_{n}^{(+)} F_{m}^{(-)} \frac{\left(u_{n, m}+e_{1}\right)\left(u_{n, m}+o_{2}\right)}{\left(e_{1}-o_{2}\right)}+F_{n}^{(-)} F_{m}^{(-)} \frac{\left(u_{n, m}+o_{2}\right)\left(u_{n, m}+o_{1}\right)}{o_{1}-o_{2}},
\end{aligned}
$$

and then that the generalized symmetry of arbitraryorder $N_{i}=q_{i}-p_{i}$ are given by (5.2)

$$
\begin{aligned}
& g_{n, m}^{(1)}=R^{\left(1, \alpha_{1}\right)} F_{n}^{(1)}\left(T_{n}^{p_{1}} W_{1}^{\left(\alpha_{1}\right)}, \ldots, T_{n}^{q_{1}} W_{1}^{\left(\alpha_{1}\right)}\right)+R^{\left(1, \beta_{1}\right)} G_{n}^{(1)}\left(T_{n}^{p_{1}} W_{1}^{\left(\beta_{1}\right)}, \ldots, T_{n}^{q_{1}} W_{1}^{\left(\beta_{1}\right)}\right), \\
& g_{n, m}^{(2)}=R^{\left(2, \alpha_{2}\right)} F_{n}^{(2)}\left(T_{n}^{p_{2}} W_{2}^{\left(\alpha_{2}\right)}, \ldots, T_{n}^{q_{2}} W_{2}^{\left(\alpha_{2}\right)}\right)+R^{\left(2, \beta_{2}\right)} G_{n}^{(2)}\left(T_{n}^{p_{2}} W_{2}^{\left(\beta_{2}\right)}, \ldots, T_{n}^{q_{2}} W_{2}^{\left(\beta_{2}\right)}\right),
\end{aligned}
$$

where $F_{k}^{(i)}\left(x_{1}, \ldots, x_{N_{i}}\right)$ and $G_{k}^{(i)}\left(x_{1}, \ldots, x_{N_{i}}\right)$ are arbitrary functions of their arguments.

\section{General solutions}

In this section we construct the general solution of the non-autonomous Hietarinta equation (1.10) using the method presented in [33, 34], which is a modification of the procedure presented 
in [21]. By general solution we mean a representation of the solution of a quad-equation (4.1) in terms of the right number of arbitrary functions of one lattice variable $n$ or $m$. Since quadequations are the discrete analogue of second order hyperbolic partial differential equations, the general solution must contain an arbitrary function in the $n$ direction and another one in the $m$ direction.

To obtain the solution we will need only the $W_{1}$ integrals we derived in Section 4 and the fact that the relation (4.2) implies $W_{1}=\xi_{n}$ with $\xi_{n}$ an arbitrary function of $n$. The equation $W_{1}=\xi_{n}$ is an ordinary difference equation in the $n$ direction depending parametrically on $m$. Then from every $W_{1}$ integral we can derive two different ordinary difference equations, one corresponding to $m$ even and one corresponding to $m$ odd. In both the resulting equations we can get rid of the two-periodic terms by considering the cases $n$ even and $n$ odd and using the definitions

$$
\begin{aligned}
& u_{2 k, 2 l}=v_{k, l}, \quad u_{2 k+1,2 l}=w_{k, l}, \\
& u_{2 k, 2 l+1}=y_{k, l}, \quad u_{2 k+1,2 l+1}=z_{k, l} .
\end{aligned}
$$

This transformation brings both equations into a system of coupled difference equations. Apply the even/odd splitting (6.1) of the lattice variables to describe a general solution we will need two arbitrary functions in both directions, i.e., we will need a total of four arbitrary functions.

We assume without loss of generality $\alpha_{1}=\beta_{1}=1$ in (4.5)

$$
\begin{aligned}
& F_{n}^{(+)} F_{m}^{(+)} \frac{\left(u_{n, m}+e_{2}\right)\left(u_{n+1, m}+o_{1}\right)}{\left(u_{n, m}+e_{1}\right)\left(u_{n+1, m}+e_{2}\right)}+F_{n}^{(+)} F_{m}^{(-)} \frac{\left(u_{n, m}+o_{2}\right)\left(u_{n+1, m}+o_{1}\right)}{\left(u_{n, m}+e_{1}\right)\left(u_{n+1, m}+o_{2}\right)} \\
& \quad+F_{n}^{(-)} F_{m}^{(+)} \frac{\left(u_{n, m}+o_{1}\right)\left(u_{n+1, m}+e_{2}\right)}{\left(u_{n, m}+e_{2}\right)\left(u_{n+1, m}+e_{1}\right)}+F_{n}^{(-)} F_{m}^{(-)} \frac{\left(u_{n, m}+o_{1}\right)\left(o_{2}+u_{n+1, m}\right)}{\left(u_{n, m}+o_{2}\right)\left(u_{n+1, m}+e_{1}\right)}=\xi_{n} .
\end{aligned}
$$

Then we treat the cases $m$ even and odd separately.

Case $m=2 l$ : In this case equation (6.2) becomes

$$
F_{n}^{(+)} \frac{\left(u_{n, 2 l}+e_{2}\right)\left(u_{n+1,2 l}+o_{1}\right)}{\left(u_{n, 2 l}+e_{1}\right)\left(u_{n+1,2 l}+e_{2}\right)}+F_{n}^{(-)} \frac{\left(u_{n, 2 l}+o_{1}\right)\left(u_{n+1,2 l}+e_{2}\right)}{\left(u_{n, 2 l}+e_{2}\right)\left(u_{n+1,2 l}+e_{1}\right)}=\xi_{n} .
$$

We can then separate the even and odd part in $n$ of equation (6.3) and apply the transformation (6.1a). We obtain the following system

$$
\begin{aligned}
& \frac{\left(v_{k, l}+e_{2}\right)\left(w_{k, l}+o_{1}\right)}{\left(v_{k, l}+e_{1}\right)\left(w_{k, l}+e_{2}\right)}=\xi_{2 k}, \\
& \frac{\left(w_{k, l}+o_{1}\right)\left(v_{k+1, l}+e_{2}\right)}{\left(w_{k, l}+e_{2}\right)\left(v_{k+1, l}+e_{1}\right)}=\xi_{2 k+1} .
\end{aligned}
$$

This system is linear and equation (6.4a) is not a difference equation, but defines $w_{k, l}$ in terms of $v_{k, l}$

$$
w_{k, l}=-\frac{\left(-o_{1}+\xi_{2 k} e_{2}\right) v_{k, l}-e_{2} o_{1}+\xi_{2 k} e_{1} e_{2}}{\left(-1+\xi_{2 k}\right) v_{k, l}-e_{2}+\xi_{2 k} e_{1}} .
$$

Inserting $w_{k, l}$ from (6.5) into equation $(6.4 \mathrm{~b})$ we obtain that $v_{k, l}$ solves the following discrete Riccati equation

$$
v_{k+1, l}=\frac{\left(-\xi_{2 k} e_{2}+\xi_{2 k+1} e_{1}\right) v_{k, l}-\xi_{2 k} e_{1} e_{2}+\xi_{2 k+1} e_{1} e_{2}}{\left(\xi_{2 k}-\xi_{2 k+1}\right) v_{k, l}-\xi_{2 k+1} e_{2}+\xi_{2 k} e_{1}} .
$$


Equation (6.6) is linearized through the Möbius transformation

$$
v_{k, l}=-e_{2}-\frac{e_{2}}{V_{k, l}}
$$

and yields the following linear equation for $V_{k, l}$

$$
V_{k+1, l}=\frac{\xi_{2 k}}{\xi_{2 k+1}} V_{k, l}-\frac{e_{2}\left(\xi_{2 k}-\xi_{2 k+1}\right)}{\xi_{2 k+1}\left(e_{1}-e_{2}\right)} .
$$

Exploiting the arbitrariness of $\xi_{2 k}$ we introduce a new arbitrary function $a_{k}$ by defining

$$
\xi_{2 k}=\frac{a_{k}}{a_{k+1}} \xi_{2 k+1}
$$

Then equation (6.8) becomes

$$
a_{k+1} V_{k+1, l}=a_{k} V_{k, l}-\frac{e_{2}\left(a_{k}-a_{k+1}\right)}{e_{1}-e_{2}} .
$$

Equation (6.10) is a total difference hence its solution is

$$
V_{k, l}=\frac{\alpha_{l}}{a_{k}}+\frac{e_{2}}{e_{1}-e_{2}} .
$$

Then inserting (6.11) into (6.7) and (6.5) we obtain the solution of the system (6.4)

$$
\begin{aligned}
& v_{k, l}=-\frac{e_{2}\left(\alpha_{l}\left(e_{1}-e_{2}\right)+a_{k} e_{1}\right)}{\alpha_{l}\left(e_{1}-e_{2}\right)+a_{k} e_{2}}, \\
& w_{k, l}=-\frac{e_{2}\left(\xi_{2 k+1} \alpha_{l} e_{1}+o_{1} a_{k+1}-\xi_{2 k+1} e_{2} \alpha_{l}\right)}{\left(a_{k+1}-\xi_{2 k+1} \alpha_{l}\right) e_{2}+\xi_{2 k+1} \alpha_{l} e_{1}} .
\end{aligned}
$$

Case $m=2 l+1$ : In this case equation (6.2) becomes:

$$
F_{n}^{(+)} \frac{\left(u_{n, 2 l+1}+o_{2}\right)\left(u_{n+1,2 l+1}+o_{1}\right)}{\left(u_{n, 2 l+1}+e_{1}\right)\left(u_{n+1,2 l+1}+o_{2}\right)}+F_{n}^{(-)} \frac{\left(u_{n, 2 l+1}+o_{1}\right)\left(o_{2}+u_{n+1,2 l+1}\right)}{\left(u_{n, 2 l+1}+o_{2}\right)\left(u_{n+1,2 l+1}+e_{1}\right)}=\xi_{n} .
$$

We can then separate the even and odd part in $n$ of equation (6.13) and apply the transformation (6.1b). We obtain the following system

$$
\begin{aligned}
& \frac{\left(y_{k, l}+o_{2}\right)\left(z_{k, l}+o_{1}\right)}{\left(y_{k, l}+e_{1}\right)\left(z_{k, l}+o_{2}\right)}=\frac{a_{k}}{a_{k+1}} \xi_{2 k+1}, \\
& \frac{\left(z_{k, l}+o_{1}\right)\left(y_{k+1, l}+o_{2}\right)}{\left(z_{k, l}+o_{2}\right)\left(y_{k+1, l}+e_{1}\right)}=\xi_{2 k+1},
\end{aligned}
$$

where we used the definition of $\xi_{2 k}$ (6.9). This system is linear and equation (6.14a) is not a difference equation, but defines $z_{k, l}$ in terms of $y_{k, l}$

$$
z_{k, l}=\frac{-a_{k} o_{2}\left(y_{k, l}+e_{1}\right) \xi_{2 k+1}+o_{1} a_{k+1}\left(y_{k, l}+o_{2}\right)}{a_{k}\left(y_{k, l}+e_{1}\right) \xi_{2 k+1}-a_{k+1}\left(y_{k, l}+o_{2}\right)} .
$$

Inserting $z_{k, l}$ from (6.15) into equation (6.14b) we obtain that $y_{k, l}$ solves the following discrete Riccati equation

$$
y_{k+1, l}=\frac{e_{1}\left(y_{k, l}+o_{2}\right) a_{k+1}-a_{k} o_{2}\left(y_{k, l}+e_{1}\right)}{-\left(y_{k, l}+o_{2}\right) a_{k+1}+a_{k}\left(y_{k, l}+e_{1}\right)} .
$$


Equation (6.16) is linearized through the Möbius transformation

$$
y_{k, l}=-o_{2}-\frac{o_{2}}{Y_{k, l}}
$$

which yields the following equation for $Y_{k, l}$

$$
a_{k+1} Y_{k+1, l}=a_{k} Y_{k, l}-\frac{o_{2}\left(a_{k}-a_{k+1}\right)}{e_{1}}-o_{2}
$$

Equation (6.18) is already a total difference, so its solution is

$$
Y_{k, l}=\frac{\beta_{l}}{a_{k}}+\frac{o_{2}}{e_{1}-o_{2}} .
$$

Then inserting (6.19) into (6.17) and (6.15) we obtain the solution of the system (6.14)

$$
\begin{aligned}
& y_{k, l}=-\frac{o_{2}\left(\beta_{l}\left(e_{1}-o_{2}\right)+a_{k} e_{1}\right)}{\beta_{l}\left(e_{1}-o_{2}\right)+a_{k} o_{2}}, \\
& z_{k, l}=-\frac{o_{2}\left(\xi_{2 k+1} \beta_{l} e_{1}-\beta_{l} \xi_{2 k+1} o_{2}+o_{1} a_{k+1}\right)}{\left(a_{k+1}-\beta_{l} \xi_{2 k+1}\right) o_{2}+\xi_{2 k+1} \beta_{l} e_{1}} .
\end{aligned}
$$

The solution of the non-autonomous Hietarinta equation (1.10) is then given by formulas (6.12) and (6.20). The four arbitrary functions are $\xi_{2 k+1}, a_{k}, \alpha_{l}$ and $\beta_{l}$. It can be checked that this is the general solution by inserting it into (1.10)

\section{Conclusions}

In this paper we presented a new approach to the Hietarinta equation (1.4). From the consideration that the Hietarinta equation possesses the property of the consistency around the cube [38], but does not possess the discrete symmetries of the square (1.3) we applied to it the Boll's construction developed in [2, 8, 9, 10, 40, 61]. The result was a seemingly new non-autonomous, two-periodic quad-equation which we called the non-autonomous Hietarinta equation (1.10). In this sense we "reconstructed" the Hietarinta equation (1.4), since from the same single-cell equation instead of using the standard embedding (1.5) we adopted a different one resulting in a different equation on the $\mathbb{Z}^{2}$ lattice.

We devoted the rest of the paper to the study of the integrability properties of this equation. The results of Sections 2-6 can be seen as a nice example of the properties that were found for the two classes of linearizable equations belonging to Boll's classifications, namely the trapezoidal $H^{4}$ equations and the $H^{6}$ equations [28, 29, 30, 31, 32, 33, 34]. Indeed we proved in Section 3 that the non-autonomous Hietarinta equation can be mapped into a particular case of the ${ }_{1} D_{4}$ equation, an equation belonging to the $H^{6}$ class. We remark that the identification of the non-autonomous Hietarinta equation (1.10) with equation (1.13) is possible only in the extended framework of the consistency around the cube given in $[2,8,9,10]$, since in order to transform the non-autonomous Hietarinta equation (1.10) into (1.13) the non-autonomous Möbius transformation (3.3) must be used. In the framework of [1] this is not possible since transformations of this kind are not allowed. Therefore the construction carried out in this paper is "necessary" to obtain this result.

In this work we prove that the problem of the embedding of single cell quad-equations possessing the consistency around the cube is crucial. As already discussed in [40] there might exist multiple embeddings preserving on the whole lattice the consistency around the cube. To understand how many and what they are is then a relevant task. We conjecture that in the procedure of extension might be essential to form bi-quadratic patterns $[8,9,10]$. To form a bi-quadratic pattern means that, given a quad-equation on the single cell (1.1), to construct an equation on 
the $\mathbb{Z}^{2}$ lattice in a way such that the bi-quadratics (1.6) pertaining to the sides of neighboring cells are of the same type. However preserving the bi-quadratic patterns might be ineffectual in the case of the $H^{6}$ due to the lack of a well-defined "black/white" assignment to the vertexes of the elementary cell.

In Section 3 we discussed, exploiting the Möbius transformation (3.3), how the non-auton̄omous Hietarinta equation (1.10) can arise as bottom equation from a triplet possessing the tetrahedron property or from a triplet without tetrahedron property. This leaves open the problem if any other known quad-equation may arise from triplet without tetrahedron property and what kind of information such non-tetrahedron triplet can give.

Finally we point out that whereas it was very easy to prove the Darboux integrability for the non-autonomous Hietarinta equation (1.10), if an analogous result holds for the Hietarinta equation (1.4) with the standard embedding (1.5) it is not known. It was conjectured in [26] that the Hietarinta equation (1.4) with the standard embedding (1.5) is Darboux integrable with first integrals of order greater than two, but technical difficulties prevent a full proof of this statement.

\section{Acknowledgements}

We would like to thank Professor Decio Levi for the many interesting and fruitful discussion during the preparation of this paper. We thank the anonymous referees for their suggestions on how to improve the paper. GG is supported by INFN IS-CSN4 Mathematical Methods of Nonlinear Physics and by the Australian Research Council through an Australian Laureate Fellowship grant FL120100094.

\section{References}

[1] Adler V.E., Bobenko A.I., Suris Yu.B., Classification of integrable equations on quad-graphs. The consistency approach, Comm. Math. Phys. 233 (2003), 513-543, nlin.SI/0202024.

[2] Adler V.E., Bobenko A.I., Suris Yu.B., Discrete nonlinear hyperbolic equations: classification of integrable cases, Funct. Anal. Appl. 43 (2009), 3-17, arXiv:0705.1663.

[3] Adler V.E., Startsev S.Ya., On discrete analogues of the Liouville equation, Theoret. and Math. Phys. 121 (1999), 1484-1495, solv-int/9902016.

[4] Atkinson J., Bäcklund transformations for integrable lattice equations, J. Phys. A: Math. Theor. 41 (2008), 135202, 8 pages, arXiv:0801.1998.

[5] Atkinson J., Integrable lattice equations: Connection to the Möbius group, Bäcklund transformations and solutions, Ph.D. Thesis, The University of Leeds, 2008.

[6] Bellon M.P., Viallet C.M., Algebraic entropy, Comm. Math. Phys. 204 (1999), 425-437, chao-dyn/9805006.

[7] Bobenko A.I., Suris Yu.B., Integrable systems on quad-graphs, Int. Math. Res. Not. 2002 (2002), 573-611, nlin.SI/0110004.

[8] Boll R., Classification of 3D consistent quad-equations, J. Nonlinear Math. Phys. 18 (2011), 337-365, arXiv:1009.4007.

[9] Boll R., Corrigendum: Classification of 3D consistent quad-equations, J. Nonlinear Math. Phys. 19 (2012), 1292001, 3 pages.

[10] Boll R., Classification and Lagrangian structure of 3D consistent quad-equations, Ph.D. Thesis, Technische Universität Berlin, 2012.

[11] Bridgman T., Hereman W., Quispel G.R.W., van der Kamp P.H., Symbolic computation of Lax pairs of partial difference equations using consistency around the cube, Found. Comput. Math. 13 (2013), 517-544, arXiv:1308.5473.

[12] Butler S., Hay M., Simple identification of fake Lax pairs, arXiv:1311.2406.

[13] Butler S., Hay M., Two definitions of fake Lax pairs, AIP Conf. Proc. 1648 (2015), 180006, 5 pages. 
[14] Calogero F., Degasperis A., Spectral transform and solitons. Vol. I. Tools to solve and investigate nonlinear evolution equations, Studies in Mathematics and its Applications, Vol. 13, North-Holland Publishing Co., Amsterdam - New York, 1982.

[15] Calogero F., Nucci M.C., Lax pairs galore, J. Math. Phys. 32 (1991), 72-74.

[16] Diller J., Dynamics of birational maps of $\mathbf{P}^{2}$, Indiana Univ. Math. J. 45 (1996), 721-772.

[17] Diller J., Favre C., Dynamics of bimeromorphic maps of surfaces, Amer. J. Math. 123 (2001), 1135-1169.

[18] Doliwa A., Santini P.M., Multidimensional quadrilateral lattices are integrable, Phys. Lett. A 233 (1997), 365-372, solv-int/9612007.

[19] Falqui G., Viallet C.M., Singularity, complexity, and quasi-integrability of rational mappings, Comm. Math. Phys. 154 (1993), 111-125, hep-th/9212105.

[20] Garifullin R.N., Gudkova E.V., Habibullin I.T., Method for searching higher symmetries for quad-graph equations, J. Phys. A: Math. Theor. 44 (2011), 325202, 16 pages, arXiv:1104.0493.

[21] Garifullin R.N., Yamilov R.I., Generalized symmetry classification of discrete equations of a class depending on twelve parameters, J. Phys. A: Math. Theor. 45 (2012), 345205, 23 pages, arXiv:1203.4369.

[22] Garifullin R.N., Yamilov R.I., Integrable discrete nonautonomous quad-equations as Bäcklund autotransformations for known Volterra and Toda type semidiscrete equations, J. Phys. Conf. Ser. 621 (2015), 012005, 18 pages, arXiv:1405.1835.

[23] Grammaticos B., Halburd R.G., Ramani A., Viallet C.M., How to detect the integrability of discrete systems, J. Phys. A: Math. Theor. 42 (2009), 454002, 30 pages.

[24] Grammaticos B., Ramani A., Viallet C.M., Solvable chaos, Phys. Lett. A 336 (2005), 152-158, math-ph/0409081.

[25] Gubbiotti G., Integrability of difference equations through algebraic entropy and generalized symmetries, in Symmetries and Integrability of Difference Equations, Editors D. Levi, R. Verge-Rebelo, P. Winternitz, CRM Ser. Math. Phys., Springer, Cham, 2017, 75-151.

[26] Gubbiotti G., Integrability properties of quad equations consistent on the cube, Ph.D. Thesis, Università degli Studi Roma Tre, 2017.

[27] Gubbiotti G., Hay M., A SymPy module to calculate algebraic entropy for difference equations and quadrilateral partial difference equations, in preparation.

[28] Gubbiotti G., Scimiterna C., Levi D., Algebraic entropy, symmetries and linearization of quad equations consistent on the cube, J. Nonlinear Math. Phys. 23 (2016), 507-543, arXiv:1603.07930.

[29] Gubbiotti G., Scimiterna C., Levi D., Linearizability and a fake Lax pair for a nonlinear nonautonomous quad-graph equation consistent around the cube, Theoret. and Math. Phys. 189 (2016), 1459-1471.

[30] Gubbiotti G., Scimiterna C., Levi D., On partial differential and difference equations with symmetries depending on arbitrary functions, Acta Polytechnica 56 (2016), 193-201, arXiv:1512.01967.

[31] Gubbiotti G., Scimiterna C., Levi D., The non-autonomous YdKN equation and generalized symmetries of Boll equations, J. Math. Phys. 58 (2017), 053507, 18 pages, arXiv:1510.07175.

[32] Gubbiotti G., Scimiterna C., Levi D., A two-periodic generalization of the $Q_{\mathrm{V}}$ equation, J. Integrable Syst. 2 (2017), xyx004, 13 pages.

[33] Gubbiotti G., Scimiterna C., Yamilov R.I., Darboux integrability of trapezoidal $H^{4}$ and $H^{4}$ families of lattice equations II: General solutions, arXiv:1704.05805.

[34] Gubbiotti G., Yamilov R.I., Darboux integrability of trapezoidal $H^{4}$ and $H^{4}$ families of lattice equations I: First integrals, J. Phys. A: Math. Theor. 50 (2017), 345205, 26 pages, arXiv:1608.03506.

[35] Hay M., A completeness study on discrete, $2 \times 2$ Lax pairs, J. Math. Phys. 50 (2009), 103516, 29 pages, arXiv:0806.3940.

[36] Hay M., A completeness study on certain $2 \times 2$ Lax pairs including zero terms, SIGMA 7 (2011), 089, 12 pages, arXiv:1104.0084.

[37] Hietarinta J., A new two-dimensional lattice model that is 'consistent around a cube', J. Phys. A: Math. Gen. 37 (2004), L67-L73, nlin.SI/0311034.

[38] Hietarinta J., Searching for CAC-maps, J. Nonlinear Math. Phys. 12 (2005), suppl. 2, 223-230.

[39] Hietarinta J., Viallet C.M., Searching for integrable lattice maps using factorization, J. Phys. A: Math. Theor. 40 (2007), 12629-12643, arXiv:0705.1903. 
[40] Hietarinta J., Viallet C.M., Weak Lax pairs for lattice equations, Nonlinearity 25 (2012), 1955-1966, arXiv:1105.3329.

[41] Joshi N., Kitaev A.V., Treharne P.A., On the linearization of the first and second Painlevé equations, J. Phys. A: Math. Theor. 42 (2009), 055208, 18 pages, arXiv:0806.0271.

[42] Lando S.K., Lectures on generating functions, Student Mathematical Library, Vol. 23, Amer. Math. Soc., Providence, RI, 2003.

[43] Levi D., Yamilov R.I., The generalized symmetry method for discrete equations, J. Phys. A: Math. Theor. 42 (2009), 454012, 18 pages, arXiv:0902.4421.

[44] Levi D., Yamilov R.I., Generalized symmetry integrability test for discrete equations on the square lattice, J. Phys. A: Math. Theor. 44 (2011), 145207, 22 pages, arXiv:1011.0070.

[45] Nijhoff F.W., Lax pair for the Adler (lattice Krichever-Novikov) system, Phys. Lett. A 297 (2002), 49-58, nlin.SI/0110027.

[46] Nijhoff F.W., Walker A.J., The discrete and continuous Painlevé VI hierarchy and the Garnier systems, Glasg. Math. J. 43A (2001), 109-123, nlin.SI/0001054.

[47] Ramani A., Joshi N., Grammaticos B., Tamizhmani T., Deconstructing an integrable lattice equation, J. Phys. A: Math. Gen. 39 (2006), L145-L149.

[48] Rasin O.G., Hydon P.E., Symmetries of integrable difference equations on the quad-graph, Stud. Appl. Math. 119 (2007), 253-269.

[49] Roberts J.A.G., Tran D.T., Algebraic entropy of (integrable) lattice equations and their reductions, arXiv:1703.01069.

[50] Russakovskii A., Shiffman B., Value distribution for sequences of rational mappings and complex dynamics, Indiana Univ. Math. J. 46 (1997), 897-932, math.CV/9604204.

[51] Sakai H., Rational surfaces associated with affine root systems and geometry of the Painlevé equations, Comm. Math. Phys. 220 (2001), 165-229.

[52] Startsev S.Ya., Darboux integrable discrete equations possessing an autonomous first-order integral, J. Phys. A: Math. Theor. 47 (2014), 105204, 16 pages, arXiv:1310.2282.

[53] Startsev S.Ya., On relationships between symmetries depending on arbitrary functions and integrals of discrete equations, J. Phys. A: Math. Theor. 50 (2017), 50LT01, 12 pages, arXiv:1611.02235.

[54] SymPy Development Team, SymPy: Python library for symbolic mathematics, 2016, http://www.sympy . org.

[55] Takenawa T., Discrete dynamical systems associated with root systems of indefinite type, Comm. Math. Phys. 224 (2001), 657-681, nlin.SI/0103016.

[56] Tremblay S., Grammaticos B., Ramani A., Integrable lattice equations and their growth properties, Phys. Lett. A 278 (2001), 319-324, arXiv:0709.3095.

[57] Veselov A.P., Growth and integrability in the dynamics of mappings, Comm. Math. Phys. 145 (1992), 181-193.

[58] Viallet C.M., Algebraic entropy for lattice equations, math-ph/0609043.

[59] Viallet C.M., Integrable lattice maps: $Q_{\mathrm{V}}$, a rational version of $Q_{4}$, Glasg. Math. J. 51 (2009), 157-163, arXiv:0802.0294.

[60] Viallet C.M., On the algebraic structure of rational discrete dynamical systems, J. Phys. A: Math. Theor. 48 (2015), 16FT01, 21 pages, arXiv:1501.06384.

[61] Xenitidis P.D., Papageorgiou V.G., Symmetries and integrability of discrete equations defined on a blackwhite lattice, J. Phys. A: Math. Theor. 42 (2009), 454025, 13 pages, arXiv:0903.3152.

[62] Yamilov R., Symmetries as integrability criteria for differential difference equations, J. Phys. A: Math. Gen. 39 (2006), R541-R623.

[63] Zhiber A.V., Sokolov V.V., Exactly integrable hyperbolic equations of Liouville type, Russian Math. Surveys 56 (2001), no. 1, 61-101. 